$\Rightarrow$ 2D MATERIALS

\title{
A new model for friction
}

Atomic force microscopy measurements and simulations have shown that friction in 2D materials decreases with an increasing number of layers, and that friction gets stronger at the onset of motion. Both phenomena

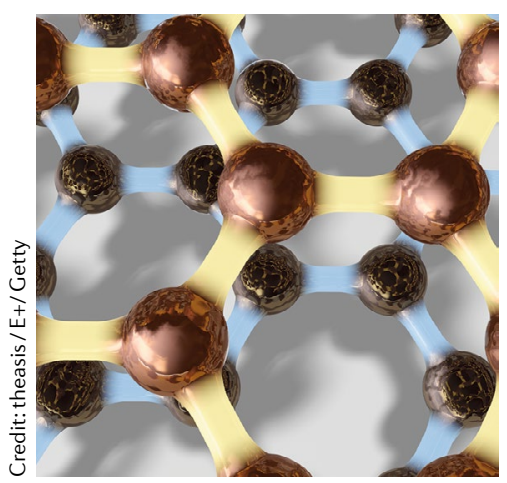

have been observed in a variety of materials, including graphene, molybdenum disulfide and hexagonal boron nitride, but different studies have reached different conclusions regarding the mechanism at work. Now, writing in Nature Communications, David Andersson and Astrid de Wijn present a simple model that explains why this was the case.

Andersson and de Wijn considered the Prandtl-Tomlinson model, which is widely used to understand friction in atomic force microscopy experiments, and added an extra degree of freedom. This new degree of freedom describes the internal dynamics of the layered material and can represent, for example, bending, shearing or other types of deformation.

Their new model unveils the dynamics of the initial friction strengthening and the dependence of friction on the number of layers, and elucidates the important role of the substrate supporting the 2D material. Andersson and de Wijn found that apparent contradictions between previous results stem from the fact that different types of deformation, as well as subtle factors, such as the quality of the contact area between the two bodies sliding against one another, can give rise to similar dynamics. Thus, different mechanisms can underpin similar friction behaviours.

Giulia Pacchioni

ORIGINAL ARTICLE Andersson, D. \& de Wijn, A. S. Understanding the friction of atomically thin layered materials. Nat. Commun. 11, 420 (2020) 